\title{
Frequency Measurement of Transient Oscillatory on LABVIEW
}

\author{
Haichao Guo \\ Department of Electrical Engineering, North China Electric Power University, NCEPU, Baoding, China \\ Email: guohaichao42130@126.com \\ Received February 2014
}

\begin{abstract}
Due to the sensitivity of the frequency measurement of power system transient oscillatory signal with respect to noise signal, a new measurement method based on Wavelet Transform and Windowed Fourier Transform is proposed. An analysis using LabVIEW on oscillatory signal containing various noise components is carried on and it is shown that the proposed method can detect the oscillatory frequency more accurately and quickly.
\end{abstract}

\section{Keywords}

Transient Oscillatory; Frequency Measurement; Wavelet Transform; Windowed Fourier Transform

\section{Introduction}

Transient oscillation refers to the abrupt bipolar change of current and voltage in the steady state condition, which is of high frequency and short duration, it often causes the damage of the electronic equipment and operating equipment insulation, brings immeasurable loss to power quality sensitive users. Power system transient oscillation is a typical problem of transient voltage disturbance, which occurs second only to voltage sag, so the study of transient oscillation is taken more and more seriously.

A detection method of power quality disturbance based on Hilbert Huang transform (HHT) of the ensemble empirical mode decomposition (EEMD) is proposed in [1], which can detect transient oscillation frequency. A short time Fourier transform based on the special frequency component is proposed, which can obtain the main frequency and amplitude of harmonic frequency component accurately in [2]. A Prony detection method based on the specific frequency band is proposed in [3], which can detect the amplitude and frequency of transient oscillation signal. But the above methods are complex and sensitive to noise, so they do not apply to the signal containing noise. A method based on Morlet wavelet-based-spectral kurtosis (SK) is proposed, but as a result of spectral kurtosis is sensitive to noise, the detection error increases with the decreasing of the noise [4].

The Windowed Fourier transform (WFT) and Continuous wavelet transform (CWT) are two tools in the time-frequency analysis. They have a significant difference. That is, the window length is fixed for WFT and proportional to the scale for CWT, which makes the frequency resolution of WFT is inferior to CWT at low frequencies and better than CWT at high frequencies [5]. 
In order to measure frequency, the main characteristic of the transient oscillation, this paper combines the CWT and WFT, which selects CWT in Long time scale and WFT in short time scale. That is, denoising to the original signal in the first, then the Binary frequency characteristic of CWT is used to calculate the percentage of each frequency band signal, so the oscillation frequency range is reduced and the optimal window is obtained, the oscillation frequency is measured using WFT finally. LabVIEW is a kind of graphical programming tools, widely used in research laboratory. The above method is proved effective using LabVIEW in this paper.

\section{The Algorithm Principle}

\subsection{Windowed Fourier Transform}

Windowed Fourier transform is defined as:

$$
F(\omega, \tau)=\int_{-\infty}^{\infty} f(t) g(t-\tau) e^{-j \omega t} d t
$$

Here, $f(t)$ is the signal to be analyzed, $g(t)$ is the window function, and $\tau$ is a translation vector.

The frequency range and accuracy of WFT are determined by the window function, and it is once established, whose shape is no longer changed. Due to the uncertainty in advance of main frequency components, scope, time and duration of transient signal, the process of adding window function exists blindness. In order to find the right point to add window, moving the window in the entire signal and making several attempts to get the right window length are needed, which bring a large amount of calculation. If the frequency scope, time and duration of the transient signal are predetermined, a very good analysis result is obtained only by adding window once.

\subsection{Wavelet Transform}

On the basis of inheriting and developing the idea of WFT time-frequency localization, while overcoming the disadvantage of the WFT fixed window size and no discrete orthogonal, Wavelet transform is suitable for transient signal analysis.

Assuming $\Psi(t)$ is square integrable function, i.e. $\Psi(t) \in L^{2}(R)$. If its Fourier transform $\Psi(\omega)$ satisfies the conditions:

$$
C_{\Psi}=\int_{R} \frac{|\Psi(\omega)|^{2}}{|\omega|} d \omega<\infty
$$

$\Psi(t)$ is called a basic wavelet or mother wavelet. After the expansion and translation of the mother wavelet $\Psi(t)$, a wavelet sequence is obtained.

Wavelet analysis technique handles Frequency domain information in band when dealing with transient signals, so you can extract characteristic signal of transient signal using its multi-resolution analysis, simply by selecting the appropriate decomposition level. This makes the main frequency transient signal include in a subband, but the frequency and amplitude corresponding to the individual harmonics cannot be obtained.

\subsection{Wavelet Thresholding Denoising}

Based on the sparseness and decorrelation of CWT, most of the wavelet coefficients are close to zero and the useful signals focus on a few frequency bands. But the noise energy is distributed uniformly, so the coefficients of the useful signal is usually greater than noise. Therefore, decomposing first, then the threshold value is set to get the useful wavelet coefficients for signal reconstruction. Generally, wavelet thresholding denoising includes the following three steps [6]-[9].

1) Wavelet decomposition. To signals containing Noisy, select the appropriate wavelet function and determine the number of layers to decompose $\mathrm{M}$, then Wavelet decomposition is carried on to the signal $\mathrm{f}$ and wavelet coefficients are obtained.

2) Thresholding. To wavelet coefficients, select threshold based on the distribution of noise to remove redundant coefficients, so the new wavelet coefficients are obtained.

3) Wavelet reconstruction. Do wavelet reconstruction using the retained wavelet coefficient after thresholding, so the original signal estimated is recovered. 


\subsection{Specific Frequency Band Fixing}

The original signal is decomposed into multiple sub-bands using wavelet multi-resolution analysis, and the frequency range of each band is obtained. The energy of each sub-band can be calculated by the following formula:

$$
E_{i}=\int\left|f_{i}(t)\right|^{2} d t=\sum_{k=1}^{n}\left|x_{i}(k)\right|^{2}
$$

where, $f_{i}$ is the signal in the sub band $i ; x_{i}$ is discrete value.

Comparing the energy of each sub-band, the sub-band carrying the most energy is get to do signal reconstruction, thereby a specific band is obtained which contains the main high-frequency components of the original.

\subsection{Determination of the Signal Center and Window Width}

Following the definition of center of gravity in mechanics, the signal center is as follows:

$$
\tau=\frac{\int_{R} t|f(t)|^{2} d t}{\int_{R}|f(t)|^{2} d t}
$$

where, $f_{i}$ is the signal in the sub band $i ; x_{i}$ is discrete value.

Then Following the definition of torques, the $1 / 2$ width of window function is:

$$
\Delta t=\sqrt{\frac{\int_{R}(t-\tau)^{2}|f(t)|^{2} d t}{\int_{R}|f(t)|^{2} d t}}
$$

By Equations (4) and (5) the window width can be obtained:

$$
\Delta \omega=2 \Delta t
$$

After the time center and window width are calculated, select the appropriate window function for WFT.

\section{The Simulation Test}

\subsection{Oscillation Waveform Generation}

Using transient disturbance model in Labview environment the transient oscillation waveform is produced, whose model is:

$$
u(t)=\sin \omega t+\alpha e^{-c\left(t-t_{1}\right)}\left[u\left(t-t_{1}\right)-u\left(t-t_{2}\right)\right] \sin \beta \omega t
$$

where, $0.1 \leq \alpha \leq 0.8, T \leq t_{2}-t_{1} \leq 3 T$, Figure 1 is the waveform.

Figure 1 constructs four cycles of $0.08 \mathrm{~s} . t_{1}=0.03 \mathrm{~s}$ and $t_{2}=0.033 \mathrm{~s}$, i.e., The oscillation starts from $0.03 \mathrm{~s}$ and continues 0.003 s. $\omega_{0}=100 \pi, \beta=60, \alpha=0.7$, i.e., The oscillation whose frequency is $3000 \mathrm{~Hz}$ and amplitude is 0.7 is produced. The sampling frequency is $10000 \mathrm{~Hz}$.

Using LabVIEW, this signal is broken down to 5 layers using multi-resolution analysis, and the type (3) is used to calculate the percentage of each frequency band signal of the total energy, as shown in Table $\mathbf{1}$.

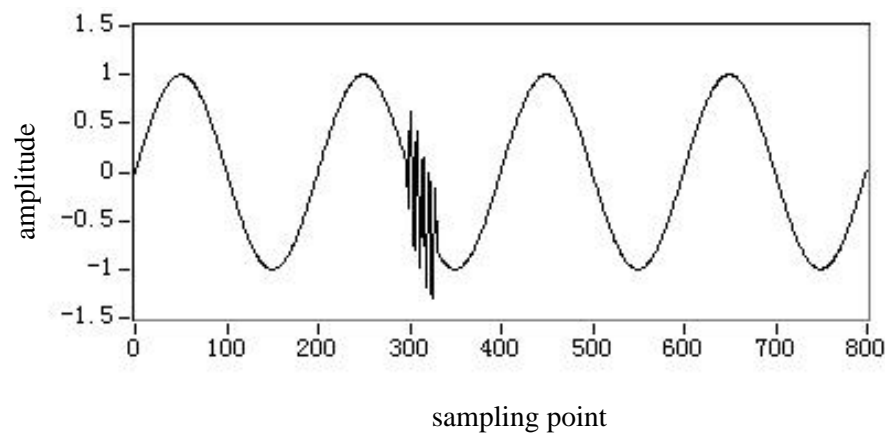

Figure 1. Transient oscillation waveform. 
Table 1. The percentage of each frequency band signal.

\begin{tabular}{ccc}
\hline Decomposition layers & The proportion of energy & Frequency range $(\mathrm{Hz})$ \\
\hline The first layer & 0.782808 & $2500-5000$ \\
The second layer & 0.0518098 & $1250-2500$ \\
The third layer & 0.0283727 & $625-1250$ \\
The fourth layer & 0.0670952 & $312.5-625$ \\
The fifth layer & 0.0699148 & $156.25-312.5$ \\
\hline
\end{tabular}

Table 1 shows that the first layer has the maximum energy, which shows that the first subspace contains the main high frequency components of the original signal. By analyzing the signal of the first layer, the high primary frequency components in original signal can be obtained.

Meanwhile, Equations (4)-(6) are used to calculate the window center $\tau=0.0315573 s, 298$ in points and window width $\Delta \omega=0.0036 \mathrm{~s}, 298$ in points.

This paper selects the Gauss window in WFT, which does not change the size of the signal, and compared with other window function, Gaussian window has the narrowest main lobe, so its frequency resolution is better than other windows.

The Frequency analysis results acquired by applying WFT are shown in Figure 2. The frequency interval

$$
\Delta f=f_{s} / N
$$

where $f_{s}$ is the sampling frequency, $\mathrm{N}$ is quantity of samples, can be distinguished as positive frequency and negative frequency after applying WFT. 1 - 17 are positive frequency, because the acquired window width will be 36 points after conversion. So While totally 36 samples are taken, frequency interval is calculated as 10,000/ $36=277.78 \mathrm{~Hz}$. Combining with the frequency range of $2500 \mathrm{~Hz}-5000 \mathrm{~Hz}$ of the first layer, the measured main oscillatory frequency should be in the frequency interval between $2500 / 277.78 \mathrm{~Hz}$ and 5000/277.78 Hz, i.e. 9 and 17. In this range, the dominant oscillatory frequency can be obtained from the above Figure 2, which is 11 $\times 277.78=3055.56 \mathrm{~Hz}$.

The results above show that the window width is 6 points more than the preset value, while the measured frequency is $55.56 \mathrm{~Hz}$ more than the preset value. The frequency deviation is $55.56 / 3000=0.01852$, i.e. $1.85 \%$. Obviously the error is small.

\subsection{Analysis of $3000 \mathrm{~Hz}$ Oscillation with $20 \%$ White Noise}

With $20 \%$ of white noise added to the oscillation waveform, the oscillation frequency is still $3000 \mathrm{~Hz}$ and other parameters are the same as shown in Figure 3.

In order to eliminate the interference of noise on the signal identification, the noise is to be removed firstly, which based on the Rigrsure threshold method and sym 7 wavelet basis.

Calculating the energy percentage of each frequency band, the results are shown in Table 2 . The data show the dominant frequency is still in the first layer.

The window center and window width obtained are: $\tau=0.03198 \mathrm{~s}, \Delta \omega=0.00237 \mathrm{~s}$. Converted to points, the windowed position is 294 points and width is 48 points.

Figure 4 is the frequency measured with WFT. As 48 samples are taken, frequency interval $\Delta f$ is 10,000/48 $=208.33 \mathrm{~Hz}$. And the frequency range of the first layer is $2500 \mathrm{~Hz}-5000 \mathrm{~Hz}$. Thus the measured main frequency of the oscillation is $2500 / 208.33 \mathrm{~Hz}-5000 / 208.33 \mathrm{~Hz}$, i.e. 12 and 24 . In this range, the main oscillatory frequency can be obtained from the above Figure 4, which is $208.33 \times 14=2916.62 \mathrm{~Hz}$ and the error is $2.78 \%$.

\subsection{Analysis of $3000 \mathrm{~Hz}$ Oscillation with $30 \%$ White Noise}

When 30\% noise added and the other parameters fixed, a percentage of the total energy of each band signal is obtained using the same process as above. The results are shown in Table 3. Table $\mathbf{3}$ shows the main frequency is still on the first layer.

The window center and window width obtained are: $\tau=0.0314544 \mathrm{~s}, \Delta \omega=0.00303 \mathrm{~s}$. Converted to points, the windowed position is 285 points and width is 60 points. 


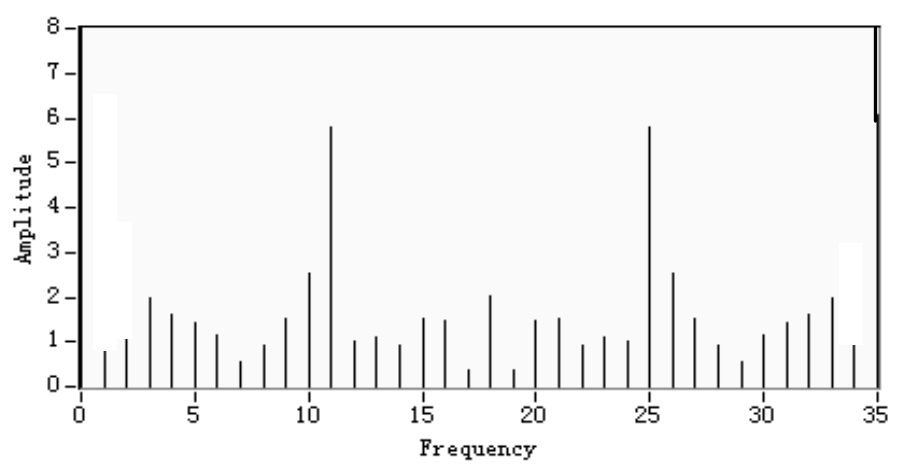

Figure 2. Transient oscillation at $3000 \mathrm{~Hz}$.

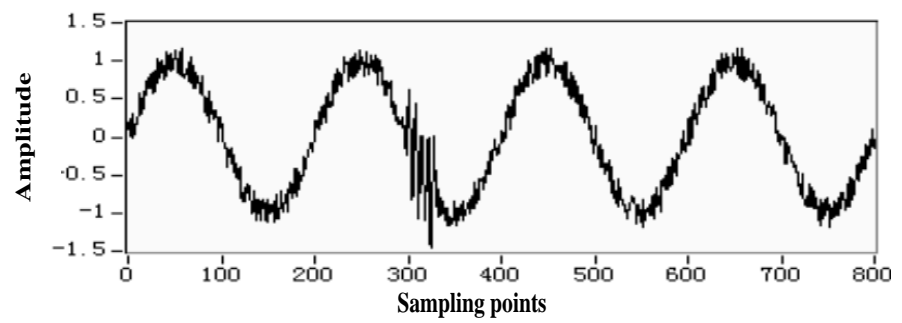

Figure 3. Transient oscillation waveform with noise.

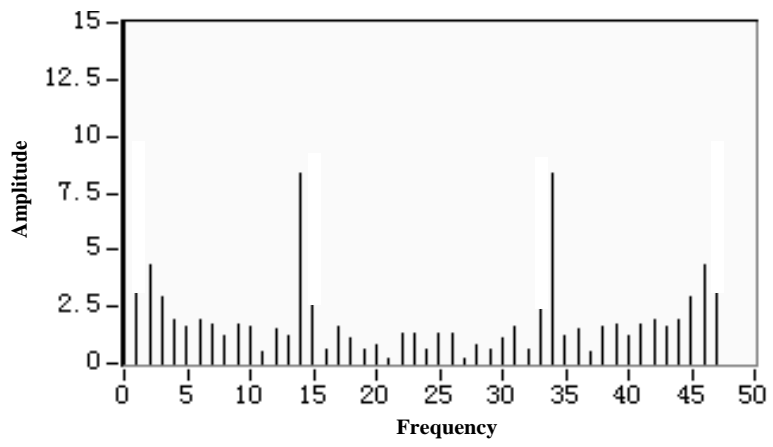

Figure 4. Transient oscillation at $3000 \mathrm{~Hz}$ after denoising.

Table 2. The percentage of each frequency band signal after denoising ( $3000 \mathrm{~Hz}, 20 \%$ noise).

\begin{tabular}{ccc}
\hline Decomposition layers & The proportion of energy & Frequency range $(\mathrm{Hz})$ \\
\hline The first layer & 0.79552 & $2500-5000$ \\
The second layer & 0.05441 & $1250-2500$ \\
The third layer & 0.0260326 & $625-1250$ \\
The fourth layer & 0.044546 & $312.5-625$ \\
The fifth layer & 0.0286896 & $156.25-312.5$ \\
\hline
\end{tabular}

Figure 5 is the frequency measured with WFT. The frequency interval $\Delta f$ is $10,000 / 60=166.67 \mathrm{~Hz}$. And the frequency range of the first layer is $2500 \mathrm{~Hz}-5000 \mathrm{~Hz}$. Thus the measured main frequency of the oscillation is $2500 / 166.67 \mathrm{~Hz}-5000 / 166.67 \mathrm{~Hz}$, i.e. 15 and 30. In this range, the main oscillatory frequency can be obtained from the above Figure 5, which is $17 \times 166.67=2833.39 \mathrm{~Hz}$.

It can be seen from the above results that window width appeared larger derivation and the main frequency measured is less than preset value $166.61 \mathrm{~Hz}$, i.e. the frequency error is $166.61 / 3000=0.0555$, which increases. 


\subsection{Analysis of $2000 \mathrm{~Hz}$ Oscillation with $20 \%$ White Noise}

When the oscillation frequency is $2000 \mathrm{~Hz}$ with $20 \%$ noise, the following results are got using the same method (Table 4).

The window center and window width obtained are: $\tau=0.0317484 \mathrm{~s}, \Delta \omega=0.00336 \mathrm{~s}$. Converted to points, the windowed position is 283 points and width is 68 points.

Figure 6 is the frequency measured with WFT. The frequency interval $\Delta f$ is 10,000/68 $=147.06 \mathrm{~Hz}$. And the frequency range of the first layer is $1250 \mathrm{~Hz}-2500 \mathrm{~Hz}$. Thus the measured main frequency of the oscillation is $1250 / 147.06 \mathrm{~Hz}-2500 / 147.06 \mathrm{~Hz}$, i.e. 9 and 17 . In this range, the main oscillatory frequency can be obtained from the above Figure 6, which is $13 \times 147.06=1911.78 \mathrm{~Hz}$. The frequency error is $88.22 / 2000=0.04411$, i.e. $4.41 \%$, which is larger than that of $3000 \mathrm{~Hz}$ with $20 \%$ noise.

\section{Conclusion}

A frequency measurement of transient oscillation signals containing noise method is combined Wavelet Transform and Windowed Fourier Transform based on LabVIEW is proposed in this paper, using which the window

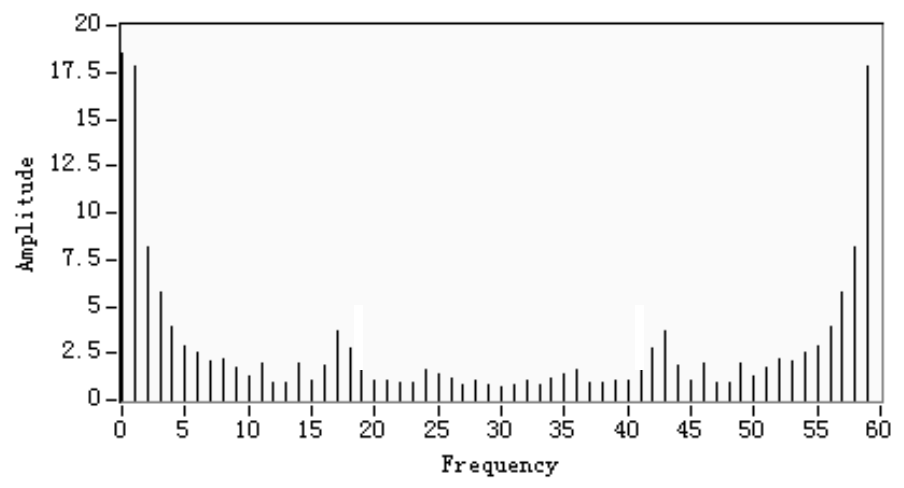

Figure 5. Transient oscillation at $3000 \mathrm{~Hz}$ after denoising.

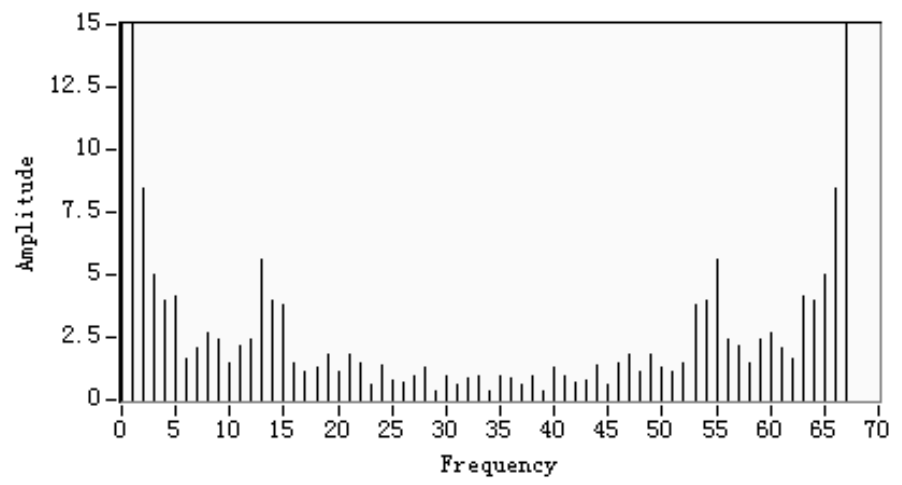

Figure 6. Transient oscillation at $2000 \mathrm{~Hz}$ after denoising.

Table 3. The percentage of each frequency band signal after denoising (3000 Hz, 30\% noise).

\begin{tabular}{ccc}
\hline Decomposition layers & The proportion of energy & Frequency range $(\mathrm{Hz})$ \\
\hline The first layer & 0.585836 & $2500-5000$ \\
The second layer & 0.0601262 & $1250-2500$ \\
The third layer & 0.0456442 & $625-1250$ \\
The fourth layer & 0.130186 & $312.5-625$ \\
The fifth layer & 0.178208 & $156.25-312.5$ \\
\hline
\end{tabular}


Table 4. The percentage of each frequency band signal after denoising (2000 Hz, 20\% noise).

\begin{tabular}{ccc}
\hline Decomposition layers & The proportion of energy & Frequency range $(\mathrm{Hz})$ \\
\hline The first layer & 0.117354 & $2500-5000$ \\
The second layer & 0.679704 & $1250-2500$ \\
The third layer & 0.0577967 & $625-1250$ \\
The fourth layer & 0.0533815 & $312.5-625$ \\
The fifth layer & 0.091764 & $156.25-312.5$ \\
\hline
\end{tabular}

length and the window position are determined quickly, and the amount of calculation is reduced effectively. Experimental results show that, while the window width and window location varies with the change of the oscillation frequency of the signal and noise levels, but always covers the whole oscillation area, so the oscillation frequency is measured within a certain error range eventually.

\section{References}

[1] Zhang, Y. and Liu, Z.G. (2011) Application of EEMD in Power Quality Disturbance Detection. Electric Power Automation Equipment, 31, 86-91. http://dx.doi.org/10.1080/028418501127346846

[2] Zhao, C.Y. and He, M.F. (2004) Short Time Fourier Analysis Based on the Special Frequency Component. Automation of Electric Power Systems, 28, 41-44.

[3] Chen, X.F. and Zhao, C.Y. (2011) The Prony Detection Method of Transient Oscillation Based on the Specific Frequency Band. Electrical Measurement \& Instrumentation, 48, 1-5. http://dx.doi.org/10.1109/9.402235

[4] Zhang, Q.G., Liu, Z.G. and Chen, G. (2013) Frequency Detection of Transient Oscillation Signal Using Morlet Wavelet Based on Spectral Kurtosis Method. Proceedings of the CSU-EPSA, 25, 1-6.

[5] Liu, L.T. and Hsu, H. (2011) Inversion and Normalization of Time-Frequency Transform. 2011 International Conference of the IEEE Conference Publications, Multimedia Technology, 26-28 July 2011, 2164-2168.

[6] Lv, R.L., Wu, T.J. and Yu, L. (2004) Performance Analysis of Threshold Denoising via Different Kinds of Mother Wavelets. Spectroscopy and Spectral Analysis, 24, 826-829.

[7] Zeng, S.Z. and Zhu, J.H. (2008) An Improved Method of De-noising in Wavelet Threshold. Ocean Technology, 27, 37-39.

[8] Fu, W. and Xu, S.C. (2006) Improved Algorithm for Threshold De-Noising in Wavelet Transform Domain. Chinese Journal of Sensors and Actuators, 19, 534-536.

[9] Wang, B., Li, J.W. and Wang, Z.F. (2011) Threshold De-Noising Method Based on Wavelet Analysis. Computer Engineering and Designs, 32, 1099-1102. 